\title{
A figural approach to the role of melodic contour in melody recognition
}

\author{
MARY C. DYSON and ANTHONY J. WATKINS \\ University of Reading, Reading, England
}

\begin{abstract}
These experiments looked at specific components of melodic contour, reversals in pitch, and nonreversals, using a short-term recognition memory paradigm. Listeners found it easier to discriminate between same and different melody pairs if changes occurred at contour reversals. This result was independent of rate of presentation, suggesting that the salience of upper and lower reversals is not attributable to a form of perceptual streaming. These results suggest an analogy between auditory and visual contours. If melodic contour is represented as a series of pitches extending over time, then reversals can be interpreted as "corners" and nonreversals as "slopes." The results are consistent with a global strategy of perceptual analysis whereby corners are more salient because they "define the figure." An alternative explanation is that the melodies are processed in a way that is similar to the processing of the intonation pattern of speech. These contour feature effects were drastically reduced when the second melody was transposed and/or following a familiarization procedure. This last result is attributed to a more detailed perceptual analysis being performed under these conditions, a process that is less influenced by contour. Therefore, while specific contour features, reversals in pitch, play a part in the recognition of untransposed novel melodies, these features are not prevalent in the recognition of transposed and more familiar sequences.
\end{abstract}

In an attempt to understand melody perception, numerous investigators have drawn upon perceptual principles derived from other modalities. Analogies have been made with the perception of visual figures, and by comparing similarities and differences between modalities the utility of general principles of perceptual organization has been explored.

This paper concentrates on the role of melodic contour in the recognition of tone sequences. Here melodic contour is broken down into components, and this represents a departure from the practice of previous workers, such as Dowling and Fujitani (1971). These authors describe contour as the overall pattern or shape of the melody, and this consists of the series of directions of pitch change (the ups and downs in pitch), disregarding the magnitude of the pitch change. The present investigation is designed to examine the perceptual significance of smaller elements of the overall contour, that is, contour components. These are examined in relation to general ideas concerning the perceptual representation of auditory sequences.

A number of previous studies have investigated the role of melodic contour in melody recognition. In these studies, either the contour remained the same

This work was supported by a grant from SERC. The authors wish to acknowledge the invaluable technical support provided by the Reading Department of Psychology, in particular that of Dave Martin. The authors' mailing address is: Department of Psychology, University of Reading, Whiteknights, Reading RG6 2AL, England. or the pattern of ups and downs was changed in a random fashion. Although these studies differ with respect to the specific hypotheses being tested, they all attempted to assess the relative contribution of contour information to the recognition of melodies.

Dowling and Fujitani (1971) looked at the contribution of contour to the recognition of transposed and untransposed melodies. Using a short-term recognition memory paradigm, they found that contour is used as a means of recognizing transposed melodies. However, when melodies are untransposed, listeners favor the use of interval information and contour effects are negligible.

Dowling (1978) used the same paradigm to separate the use of contour and scale information. Scale was defined in terms of the tonality of the melody, so that comparisons were made between tonal and atonal sequences. An atonal sequence was composed of randomly selected intervals, unrelated to a specific key. Results differed, depending on the musical experience of the listener. For the inexperienced listeners, with less than 2 years of musical training, contour was used as a means of discrimination. On the other hand, experienced listeners were able to make use of the scale or key of the melody.

Contour has also been investigated in studies that aimed to evaluate a bidimensional model of pitch. This model proposes that both tone height (the overall pitch level of a tone) and tone chroma (the position within the octave) are important in melody perception. Idson and Massaro (1978) reviewed the literature in this area. Because it is possible to con- 
found pitch height and contour (e.g., Deutsch, 1972), other studies have assessed the contribution of contour alone by looking at how contour and chroma information are used in the identification of familiar melodies (Idson \& Massaro, 1978; Kallman \& Massaro, 1979) and recently learned melodies (Massaro, Kallman, \& Kelly, 1980). The results suggested that tone chroma alone is not a sufficient cue for identification and must be accompanied by contour information. Contour and chroma together contribute to accurate identification of melodies. Contour alone can be used to identify a melody only if listeners have some knowledge of the set of tunes from which to select their answers. This is because a number of different melodies may share the same contour.

These investigations have all treated contour as the overall pattern of pitch changes. In this paper, we look at the details of contour, specifically the reversals and nonreversals in the direction of pitch. This requires a division of the overall contour of a 15note melody into overlapping sequences of 3 notes each (Figure 1). A reversal is the middle note of a 3note sequence in which the third note moves towards the first. A nonreversal occurs when the third note moves away from the first. The immediate context of each note must always be taken into account in the definition of the contour component, that is, reversal or nonreversal. This may be described as an investigation of the figural properties of melody.

This paper offers an analogy between visual and auditory representations based on apparent similarities in perceptual organization across modalities. Some Gestalt principles of organization are discussed in relation to this analogy.

Such a discussion of tone sequences as auditory figures requires some form of translation between the visual and auditory domains. This may be achieved by regarding the visual abscissa as auditory time, and the visual ordinate as the pitch height of the note. This idea has intuitive appeal because written music is traditionally notated in a similar manner, with series of notes extending from left to right; melodic

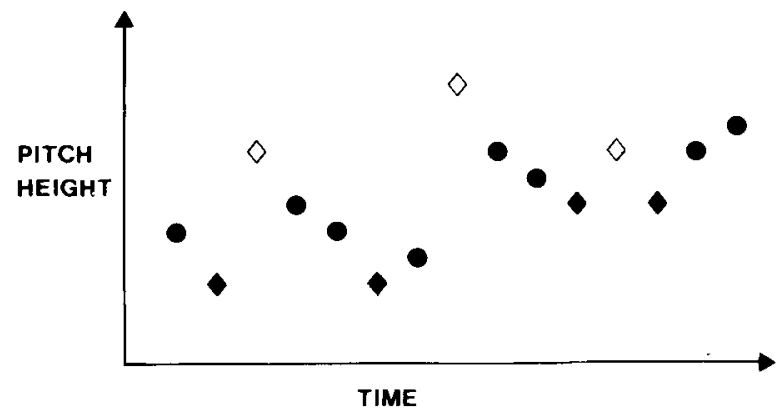

Figure 1. Contour reversals (diamonds) and nonreversals (circles) of a 15-note sequence. Unfilled diamonds represent upper reversals, and filled diamonds, lower reversals. contour then describes up and down movement over time. There is some support for the perceptual significance of such a translation: Davies and Jennings (1977) asked subjects to reproduce contour in this manner by drawing the "shape" of a familiar tune. They found that both musicians and nonmusicians were able to produce this form of representation, although musicians were more accurate.

Analogies between auditory and visual representations are not new: Ortmann $(1926,1933)$, Miller and Heise (1950), Heise and Miller (1951), and Divenyi and Hirsh (1978) used such an analogy to describe the properties of tonal patterns. Ortmann used visual representations of the outlines or contours of short tonal sequences to argue that the points that stand out for the eye are exactly those which stand out for the ear. He further proposed that the acuteness of the pitch angle determines the degree of emphasis. This angle is located at a change in pitch direction. Similarly, Heise and Miller (1951) discussed auditory sequences in terms of their shape as defined above. They maintained that a close relationship exists between the perceived continuity of an auditory pattern and the corresponding visual representation.

Some of the figural properties of auditory patterns have been investigated by testing the "validity of visually derived principles as they might apply to hearing" (Divenyi \& Hirsh, 1978, p. 1369). The superior identification of unidirectional pitch changes has been discussed in terms of the Gestalt principle of "good continuation," drawing a parallel with visual forms (Divenyi \& Hirsh, 1974, 1975). A further Gestalt rule associated with tone sequences is the "principle of proximity": a rapidly presented tone sequence may form separate auditory streams on the basis of pitch proximity. Various examples of this phenomenon have been reported (e.g., Miller \& Heise, 1950; van Noorden, 1975). This sort of grouping is illustrated by distortions in judgments of temporal order and has been labeled "primary auditory stream segregation" (Bregman \& Campbell, 1971). This pitch grouping effect has also been observed by Deutsch (1978a), who reports that pitch identification is better if intervening distractor tones are proximally related by pitch.

Two possible explanations for this form of perceptual organization have been proposed (Bregman, 1978). The first regards segregation as the breakdown of a mechanism that normally follows the order of a sequence. The alternative view is that segregation is an achievement of perception that allows the listener to separate complex sounds into meaningful parts. A number of streams are formed and components are allocated to different streams. Stream membership depends on competition between alternative groupings and is not fully explained by the degree of frequency separation. There appears to be competition 
for membership of a stream, and this is influenced by relationships between all the components (Bregman, 1978). The speed of the tone sequence is also an important influence on this type of grouping: Van Noorden has shown that rate of presentation interacts with the size of the frequency difference in twotone sequences. As rate decreases, a sequence with large frequency separations between the tones is likely to be perceived as a single coherent group, but at faster rates the sequence perceptually separates into a number of pitch groups.

This form of perceptual grouping demonstrates the utility of perceptual analogies between auditory and visual representations. That is, notes of a similar pitch height would be represented visually as being closer together at higher speeds than at lower speeds. Bregman and Achim (1973) provided a visual analogue of auditory stream segregation: at high speeds, the apparent motion of an illuminated dot splits into two substreams on the basis of positional grouping. Thus, common organizational principles may usefully be applied to both auditory and visual events.

Although various authors have made use of analogies between auditory patterns and visual figures, details of the correspondence have not been investigated. This paper aims to look at the components of melodic contour in greater detail. Findings from visual form perception serve as a guide for appropriate questions about the figural properties of melodic contour. This approach was adopted by Divenyi and Hirsh (1978), who look at the effects of transposition, distortion, and embedding of auditory figures. However, auditory research offers very little guidance, because researchers have tended to treat contour as a single global property of melodies. Exceptions to this are Ortmann (1926) and Thomassen (1982), whose work will be discussed in greater detail below.

A basic question appropriate to both modalities concerns the identification of perceptual characteristics important for the recognition of a figure. One view of the aim of a theory of visual pattern recognition is that it should be able to specify "distinctive attributes" or "features" (Ward \& Wexler, 1976). Some workers have attempted to identify those properties of features which contribute to their selection. If features enhance perceptually important differences between environmental events, then feature extraction makes for economical processing (Howard $\&$ Ballas, 1981). The important features may therefore differ from task to task (Getty, Swets, Swets, \& Green, 1979).

However, none of the above authors provide general guidelines for identifying the important features in a given situation. Attneave (1954) attempted to do this by using Gestalt principles of organization and putting these into the more quantitative framework of information theory. The points of high informa- tion value are said to be the angles or points of intersection, and these were thought to provide the most economical description of a figure.

Applications of information theory in this area are problematic (Green \& Courtis, 1966). One difficulty lies in objectively defining the elements of the figure to which the informational values are attached. Nevertheless, a study by Baker and Loeb (1973) provides some support for the importance of corners in visual perception. Their observers fixated longer on corners of patterns than on other parts and rated these sections as important for the purposes of identification.

An attempt has been made to formally describe the perceptual processes involved in visual discrimination tasks (Kroll \& Hershenson, 1980). This twostage model begins with a global comparison; it is followed by additional analytic processing if the discrimination is difficult and if there is sufficient time available. This preference for global information has been demonstrated in a recognition memory paradigm: observers retain the outline of a figure, whereas extra detail, immaterial to the global shape, is lost (Rock, Halper, and Clayton, 1972).

Thus, a theory governing the perceptual organization of visual figures might prove useful in the investigation of the figural aspects of melody such as melodic contour. Findings from visual form perception suggest that some elements of the overall contour may be more salient than others. Therefore, breaking down contour into a series of elements or components appears to be a useful approach. By referring to visual work, the problem of defining elements is circumvented. It seems reasonable to look for features of melodic contour analogous to the visual features proposed by Attneave (1954).

Two authors have dealt with specific features of auditory contours. Ortmann (1926) discussed absolute and relative factors which determine the salience of notes in a melody. The highest and lowest notes were included among the absolute factors. This salience was also extended to local pitch extremes, that is, contour reversals, or corners. However, these descriptions were based solely on observations. An experimental investigation carried out by Thomassen (1982) looked at the factors responsible for melodic accentuation. In three or four note motifs, the accent comes on the note following a frequency change. When a further change in frequency in the opposite direction occurs, the accent falls on a corner (i.e, on a contour reversal). Thus, there appears to be some correspondence between the salient aspects of visual and auditory contours.

Thomassen assumed that the accenting role of frequency changes could be extended to pitch changes in general. Comparisons can be drawn between melody and the pitch contour of speech, the latter being provided by changes in the fundamental fre- 
quency of the voice. These changes have been shown to be powerful cues to accent perception in Dutch speech (Cohen \& t'Hart, 1967). Cues to the location of stressed syllables in English have also been investigated: various workers have demonstrated that pitch is a sufficient cue for the perception of stress (Bolinger, 1958; Faure, Hirst, \& Chafcouloff, 1980; Fourcin, Rosen, Moore, Douek, Clarke, Dodson, \& Bannister, 1979; Fry, 1958; Lieberman, 1960; Morton \& Jassem, 1965). In view of the perceptual salience of these changes in speech, Thomassen argued that there was no reason why they should be less effective in music. It has also been shown that the boundary between main and embedded clauses is often characterized by a fall-rise pitch movement (Cooper \& Sorenson, 1977). These findings suggest that corners, or changes of direction, have general perceptual importance and may serve as features.

This paper examines the generality of contour features when physical parameters such as note range and stimulus waveform are varied. Alternative explanations for the salience of particular components are considered, such as the loudness variations which accompany changes in pitch. By varying the range of notes, the possible contribution of absolute and relative pitch differences can be evaluated. Finally, this investigation of features is extended to sequences that are made more familiar through repetition, and to transposed melodies. This permits a comparison of the perceptual processes involved in two quite different situations. In the first case, the listener is presented with the novel sequence only once, and in the second, the listener is given some familiarization with the melody in the course of the experiment.

\section{GENERAL METHOD}

A computer program generated a unique set of tone sequences for each listener. An attempt was made to approximate certain aspects of melodies found in Western music. This was done by using a random-number generator in conjunction with probabilistic biases in favor of certain notes and intervals. The perceived melodiousness of these sequences has been psychophysically scaled by Watkins (1981a, 1981b, 1981c, 1982). These results demonstrated that this procedure is effective in producing relatively close perceptual approximations to melody. This method of stimulus generation avoids the problems inherent in selecting a stimulus set from familiar melodies. It is unnecessary to ensure that listeners are equally familiar with all sequences (as all are novel) and the results can be more readily generalized to other tone sequences.

Melodies were generated note by note, proceeding from left to right. There were three constraints governing the selection of next notes, and further constraints were applied to control the number of contour reversals (change in the direction of pitch) and nonreversals (unidirectional pitch change).

A keynote was selected at random from the range $\mathrm{C}=261.6 \mathrm{~Hz}$ $\pm \mathbf{5}$ semitones and this note was used as the initial note of the sequence. The remaining notes were then pseudorandomly selected from a two-octave range around the keynote. ${ }^{1}$ Weightings were applied so that notes had different probabilities of occurrence. These weightings reflected the combination of the three different types of factors.

The rationale for the first factor (DQ) derives from LonguetHiggins $(1976,1978)$, who observed that a large number of Western melodies follow certain constraints on the change in the sharpness value between successive notes. This value is the number of fifths between any note and the keynote (a fifth being 7 semitones in the equal-temperament tuning system). In order to understand this idea, it is helpful to recall the progression of fifths in which the 12 notes of the octave are arranged so that adjacent notes are one fifth apart. The $q$ value of each note may then be found by counting along from the keynote. Below is shown this arrangement along with the associated value of $q$ for the case in which the keynote is $C$.

$\begin{array}{lllllllllllll}\text { note: } & \mathrm{Db} & \mathrm{Ab} & \mathrm{Eb} & \mathrm{Bb} & \mathrm{F} & \mathrm{C} & \mathrm{G} & \mathrm{D} & \mathrm{A} & \mathrm{E} & \mathrm{B} & \mathrm{F} * \\ \text { q value: } & -5 & -4 & -3 & -2 & -1 & 0 & 1 & 2 & 3 & 4 & 5 & 6\end{array}$

Notice that possible ambiguity is avoided by restricting $q$ to the range -5 through +6 .

One constraint imposed on the tone sequences made a change in $q$ of less than 6 more likely to occur than changes greater than 6 . Also, if the change in $q$ in one interval was greater than 5 , then the changes in $q$ in adjacent intervals were less than 6 . Thus, a melody swinging here and there over the $q$ dimension will be given a center of gravity or tonal center (Lerdahl \& Jackendoff, 1977) corresponding to the keynote of the melody. Furthermore, if an interval was an ascending semitone and the $q$ value of the second note was $2,3,4$, or 5 , then the $q$ value of the first note was changed to 5 plus that of the second note. This last rule avoided the false perception of continuous modulation in ascending chromatic runs.

For the $Q$ factor, the seven $q$ values of the diatonic major scale $(-1$ through +5$)$ were more likely to occur (see, e.g., Burns \& Ward, 1982, for a rationale).

Finally, the DP factor reduced the probability of larger intervals, ones greater than 5 semitones (see, e.g., Deutsch, 1978b, for a rationale).

Two methods were used to implement contour constraints. In Experiments $1,2 a$, and $2 b$, the contour was constructed from a combination of three types of elements (Figure 2) and stored as a pattern of intervals. The direction of the interval selected was then compared with the stored version. If the two agreed, this next note was appended to the sequence and became the current note. If the direction was.reversed, the probability weightings were resampled and the procedure repeated until a suitable note was found. This became too restrictive when the note range was limited to one octave, so an alternative procedure was adopted and used for the remaining experiments. This second method allowed a complete sequence to be generated, based on the above probability weightings, and this was checked for a criterion number of contour reversals and nonreversals. If the sequence failed to meet these requirements the whole process was repeated until a suitable sequence was produced.

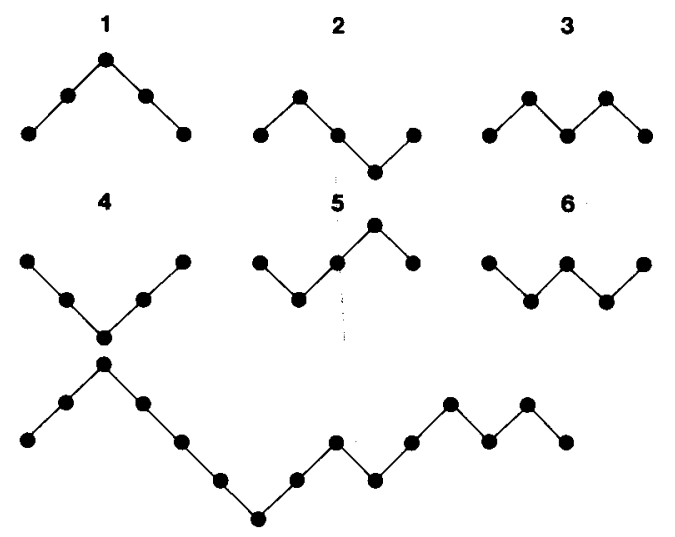

Figure 2. Three types of contour and their inverted forms used In Experiments 1 and $2 a$ and 2b. An example of a typleal contour b given below, formed by combining numbers 1,5 , and 3 . 
Each trial consisted of two tone sequences, both 15 notes long, separated by a 2-sec gap. Trials were divided into signal trials (where "same" was the correct response) and noise trials ("different" was correct). The first tone sequence of each type of trial, whether signal or noise, was generated in an identical manner, in the way described.

In Experiments $1 \mathrm{a}$ and 1b, in which changes were made to either reversals or nonreversals, four notes were changed in each noise trial. The following experiments ( $1 \mathrm{c}$ to $3 \mathrm{~b}$ ) broke reversals down into upper and lower reversals. Consequently, the number of possible changes was reduced to three as the total number of reversals in some contours was only six. By limiting to three the number of changes made, it was possible to standardize the generation of the first sequence across different noise trials. Thus, the properties of the initial melody of a trial were not influenced by the nature of the changes made to the second melody, that is, whether changes were made to upper or lower reversals or to nonreversals. Hence, any differences between the three contour components were not confounded with characteristics of the particular melodies generated on those trials. In trials in which the second melody was different, certain notes were selected for change. The selection was random, with the following exceptions: changes to the first and last notes were not permitted and changes were not made to successive notes. The latter restriction guaranteed that each new note produced two new intervals.

The note selected for change was replaced by a new note, which was generated in the same way as the original note, that is, the three constraints described above were obeyed in choosing an alternative candidate. Thus, the new note was again likely to favor the diatonic scale and form an interval with a relatively small jump in pitch and in $q$ value. Although the process of generating the new note ensured that the interval preceding this note met these criteria, the following interval was not similarly constrained. Therefore, a check was introduced to ensure that the interval formed with the following note was also likely to have been generated under the given constraints. Hence, the process of generating a different melody did not destroy the melodiousness of the sequences.

Certain restrictions were also necessary to prevent the contour from changing within a different trial. This was achieved by setting an upper and lower limit on possible new notes. These limits were defined by the notes on either side of the candidate for change or target $(T)$ and the corresponding intervals. For nonreversals, this was relatively simple as the two adjacent notes were used as the extremes of the range of possible new notes. Therefore, it was necessary only to take into account the position of the adjacent note in relation to the target, that is, whether this note was higher or lower in pitch. If the note was higher, the interval was labeled upper interval (Iu), and if the note was lower, the interval was labeled lower interval (Il). However, notes situated at contour reversals do not provide such a convenient set of upper and lower limits. This is because adjacent notes will both be above the pitch of the target for a lower reversal or both be below the pitch of the target for an upper reversal. Therefore, to ensure that the degree of change was equal for both nonreversals and reversals, the size of intervals was taken into account rather than the direction of intervals. The larger ("bigger") interval was described as Ib and the smaller interval was described as Is. Thus, the limits of change were defined for each component in terms of the target and adjacent intervals, with all intervals measured in semitones. ${ }^{2}$

$\begin{array}{ll}\text { Nonreversals } & \begin{array}{l}\text { upper limit }=\mathbf{T}+\mathrm{Iu}-1 \\ \text { lower limit }=\mathbf{T}-\mathrm{Il}+1\end{array} \\ \text { Upper reversals } & \begin{array}{l}\text { upper limit }=\mathrm{T}+\mathrm{Ib}-1 \\ \text { lower limit }=\mathrm{T}-\mathrm{Is}+1\end{array} \\ \text { Lower reversals } & \begin{array}{l}\text { upper limit }=\mathrm{T}+\mathrm{Is}-1 \\ \text { lower limit }=\mathrm{T}-\mathrm{Ib}+1\end{array}\end{array}$

The difference in semitones between the old and new notes varied within the limits described. Therefore, any perceptual differences between the different types of components cannot be attributed to differences in the number of semitones altered.

In Experiments 1 and 2, a different tune was heard on each trial. The standard rate of presentation was 4 notes $/ \mathrm{sec}$, with each note lasting $25 \mathrm{csec}$. Some other rates were included in the first experiment.

A PDP-8 computer synthesized the tunes and controlled the experiment, computing a different order of signal and noise trials within each block. A different random order was also generated for each listener. After playing a pair of tone sequences, the computer waited for the listener's response and then proceeded with the next trial. The listener responded along a 4-category rating scale ranging from "sure same," through "same" and "different," to "sure different." A C.R.O. display told the listener whether he or she was right or wrong and what the correct answer was. Sure and not sure were treated as equivalent for the purposes of this feedback.

The stimuli used in Experiment 1 were produced by varying the interpulse interval of trains of unipolar pulses which were $300 \mu \mathrm{sec}$ wide and extended over 9 bits of a 10-bit D-to-A converter. These pulse trains produced a monotonic increase of level with pitch. This was measured using a Brüel and $\mathrm{Kjaer}$ true rms voltmeter, Type 2425 , and found to be $7.7 \mathrm{~dB}$ from the bottom of the note range to the top, across the earphone connection. In Experiment 2, this waveform was compared with square waves where different pitches were produced by varying the period of the signal. These stimuli differed from pulse trains in having only odd harmonics and $\mathbf{a}$ fall-off in the amplitude from lower to higher harmonics. This gave rise to stimuli of equal intensity throughout the pitch range and these stimuli were used throughout the succeeding experiments.

For both types of stimuli, high frequencies were attenuated by a Kemo VBF $/ 8$ low-pass filter at a rate of $48 \mathrm{~dB}$ per octave above the $2-\mathrm{kHz}$ cutoff frequency. The note frequencies were tuned to the equal temperament values with a maximum error of $0.4 \%$. Listeners were seated in a soundproof booth and received diotic presentation via Sennheiser HD414 headphones. Threshold was measured for one normal-hearing listener, and sensation level was set at approximately $30 \mathrm{~dB}$ in each ear. The low listening level and low-pass filtering avoided the rather unpleasant auditory aftereffects associated with prolonged listening to pulse trains. System hum and noise were below threshold.

Twenty listeners were recruited for each experimental condition except where otherwise stated.

The data were analyzed by the PDP-8 at the end of each listener's session. A nonparametric assessment of the area under the ROC, $\mathrm{p}(\mathrm{A})$, served as the discrimination index (McNicol, 1972).

\section{EXPERIMENT 1}

This experiment examined figural properties of melodic contour by asking whether certain parts of the contour were perceptually more salient than others. There is some evidence from both visual and auditory literature which suggests that corners may serve as features in the recognition of contours. These studies were discussed in detail in the introduction. As contour reversals appear to be analogous to corners in the visual modality, there is reason to suppose that changes to melodic contour may be more likely to be detected at reversals than at nonreversals.

An alternative explanation for the salience of particular elements of a sequence stems from experiments on auditory stream segregation referred to in 
the introduction. As the context of notes influences the perceptual organization of tone sequences, certain groupings of notes may not be accounted for by frequency separation alone. Thus, although streams of upper and lower contour reversals may be formed, the greater competition for alternative groupings of nonreversals may prevent the formation of a perceptual stream. This competition can be attributed to the position of nonreversals, midway between the two extremes. This ambiguity of organization may account for any observed enhancement of salience for reversals over nonreversals.

One way of examining the appropriateness of this explanation is to vary the rate of presentation of the melodies. The literature suggests that a faster rate will enhance separation, whereas a slower rate encourages the percept of a single coherent stream of notes. This experiment tested the prediction that there would be greater differences between discriminability indices for reversals and nonreversals at fast rates that at slower rates. However, if this prediction was confirmed, this would not provide conclusive evidence for the occurrence of streaming; alternative interpretations were available. ${ }^{3}$ Nevertheless, this manipulation might still be informative: if rate had no effect on the salience of particular contour components, then stream segregation (as described by, e.g., Bregman \& Campbell, 1971) would not appear to be responsible for the results obtained. But, at the same time, we could not discount the contribution of some form of grouping of perceptually similar features (e.g., reversals in pitch) that was not affected by rate.

A more detailed investigation was also made of the perceptual salience of contour reversals by comparing upper and lower reversals. The rate of presentation was again varied to include a different rate and to observe any possible effects on these two components.

Listeners were played pairs of tunes and required to discriminate between same and different pairs. Comparisons were made between changes to reversals and nonreversals and changes to upper or lower reversals. Rate of presentation was also varied. If perceptually salient aspects of melodic contour existed, could they be manipulated by rate of presentation? If rate had no effect, then an explanation in terms of features of melodic contour might be the most parsimonious account of the findings.

\section{Method}

The experiment was divided into three parts with three independent groups of listeners. Trials were divided into blocks with short breaks in between. Each block contained a randomized order of signal (same) and noise (different) trials, with equal numbers of each of the two types of noise trial.

(a) Reversals and nonreversals. Twenty listeners received a total of 120 trials in five blocks. There were 8 signal and 16 noise trials within each block. The second tune of a noise trial contained four changes at contour reversals or at nonreversals. Reversals included upper reversals, in which an ascending interval was followed by a descending interval, and lower reversals, in which the opposite applied.

(b) Rate by reversals and nonreversals. Three rates of presentation were used, which required 180 trials. These were divided into 6 blocks, with two blocks at each rate. Listeners heard two consecutive blocks at each rate, and the order of rates was completely counterbalanced by using 24 listeners. Each block contained 20 noise and 10 signal trials. A slow rate, using notes of $35 \mathrm{csec}$ duration, and a fast rate of $10 \mathrm{csec}$ were added to the medium rate of $25 \mathrm{csec}$ used in the first part of the experiment.

(c) Upper and lower reversals by rate. Listeners received six blocks of trials, each containing 14 noise and 7 signal trials. A total of three changes were made to either upper or lower contour reversals. Each block was played at either 4 notes/sec ( $25 \mathrm{csec} /$ note) or $15 \mathrm{csec}$, with rate alternating between blocks. The order of rates was counterbalanced across subjects.

\section{Results and Discussion}

Figure 3 compares the results for reversals and nonreversals and the effect of rate on these components. Figure 4 shows the results of changes to upper and lower reversals at two rates. The discrimination index $p(A)$ obtained for each listener for each condition was transformed [ 2 arcsin root $p(A)]$ into a form suitable for parametric statistical analysis (McNicol, 1972).

(a) An analysis of variance revealed a significant difference between reversals and nonreversals $[F(1,63)=14.723, p<.001]$. This result shows that when different melodies were generated by changing contour reversals, these were more easily discriminated from an exact repetition than if changes were made to nonreversals.

(b) A two-way analysis of variance on the transformed scores revealed two main effects, rate $[F(2,115)$ $=8.232, \mathrm{p}<.001]$ and contour $[\mathrm{F}(1,115)=38.232$, $\mathrm{p}<.001]$. The faster rate produced a lower level of

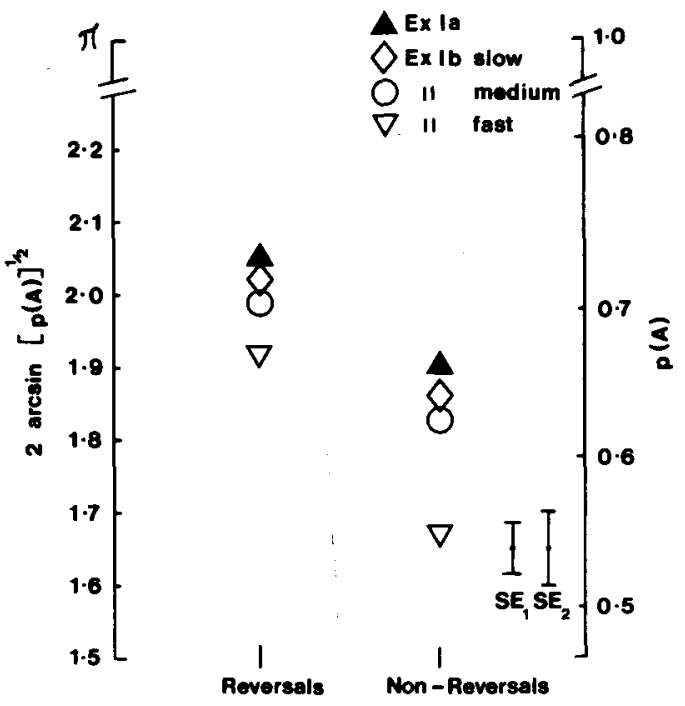

Figure 3. Results of Experiment 1a (filled triangles) and Experiment $1 \mathrm{~b}$ (unfilled symbols), means across listeners and standard errors of within-listener differences. ${ }^{4} \mathrm{SE}_{1}$ refers to Experiment $1 \mathrm{a}$ and $\mathrm{SE}_{2}$ to the interaction between contour component and rate in Experiment 1b. 


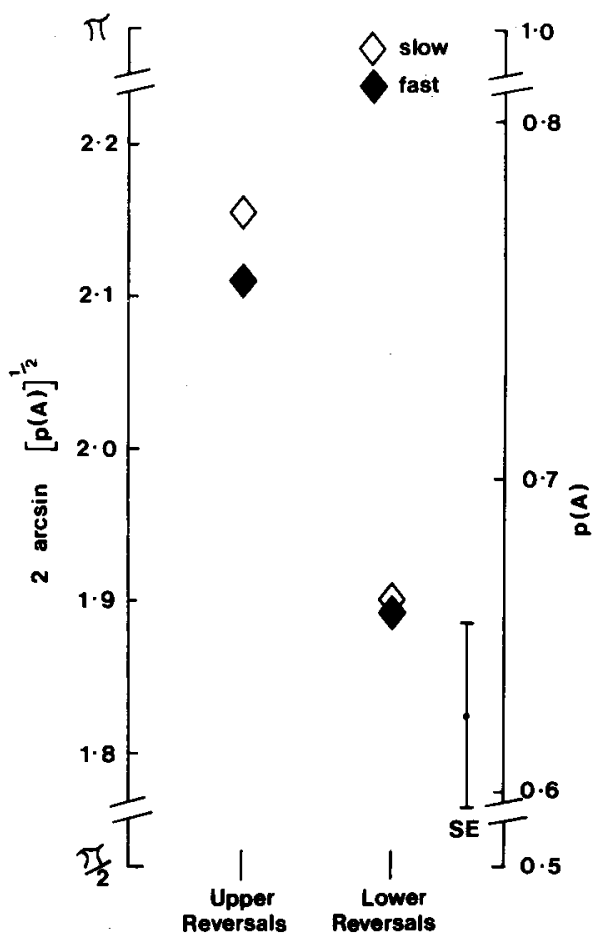

Figure 4. Results of Experiment 1c; across listener means for two rates of presentation with standard error of contour component $x$ rate interaction.

performance, but there was no interaction between contour and rate. The significant difference between reversals and nonreversals replicates Experiment 1A.

(c) A two-way analysis of variance gave only one significant $F$ ratio, a main effect of upper versus lower reversals $[F(1,57)=30.013, p<.001]$. Discrimination was easier when changes were made to the upper contour reversals than when they were made to lower reversals. The perceptual differences between the contour components were found to be independent of rate of presentation.

The results of this experiment demonstrate the perceptual salience of reversals of contour. The hypothesis that auditory stream segregation might account for differences between contour reversals and nonreversals was not confirmed. Rate influenced only the overall level of performance, but differences between the components of contour were unaffected. The lower discrimination scores with the fast rate (notes of $10 \mathrm{csec}$ duration) may be attributed to a lack of familiarity with faster sequences and problems in "following" the notes. This is supported by the lack of impairment when a rate of $15 \mathrm{csec} /$ note was used. These results support the idea that corners of melodic contour act as features and are perceptually more salient than the intervening notes.

The results of Experiments $1 \mathrm{a}$ and $1 \mathrm{~b}$ demonstrate that reversals are more salient than nonreversals.
However, the superiority of upper reversals over lower reversals raises the question of whether this result can be attributed to the greater salience of upper reversals alone. If so, it might be possible to account for the results quite simply by the hypothesis that relatively higher pitched notes are more salient, perhaps due to intensity differences: upper reversals are generally higher in pitch than nonreversals, which in turn are usually higher than lower reversals. If this hypothesis is correct, then nonreversals should be more salient than lower reversals.

However, a comparison of Figures 3 and 4 suggests that this is not the case. The mean scores for lower reversals exceed those for nonreversals at comparable rates of presentation. But the data from this experiment do not allow us to compare performance across contour components within the same listener. A more sensitive test of the relative salience of the three components, upper, lower and nonreversals, is provided by the within-listener comparisons of the following experiment.

\section{EXPERIMENT 2}

This experiment examined the relative salience of upper and lower contour reversals and nonreversals by comparing listeners' performance across all three components. This within-listener comparison is consistent with the type of comparisons previously made between contour components. This particular design provides a means of deciding between alternative explanations for the reversal over nonreversal advantage, suggested by the previous experiment. The significance of contour reversals may be due to inherent properties of reversals and therefore shared by both lower and upper reversals. Alternatively, the result obtained may be due to upper reversals alone or simply to relative pitch height and corresponding intensity differences.

Due to the importance attached to comparisons between upper reversals (generally higher notes) and lower reversals (generally lower notes), two types of stimulus waveform were used, pulse trains and square waves. Pulse trains allow a variation in the level of notes, depending on pitch, so that notes of a higher pitch are also of greater intensity. However, the square-wave stimuli included in this experiment maintain a constant level throughout the pitch range. Therefore, we are able to disentangle any effects of intensity differences from the effects of contour components.

Other factors confounded with different parts of melodic contour were also manipulated in this experiment. Absolute pitch height and relative pitch differences were varied by generating sequences using tones selected from either a one- or two-octave note range. If the size of the note range interacted with ef- 
fects of contour components, then relative and/or absolute pitch height must be a factor influencing perceptual saliency. The absolute pitch of upper reversals is generally higher with the larger note range than with the smaller range. The relative differences in pitch between upper and lower reversals is also greater, on average, in the larger (two-octave) range. In addition, larger intervals between adjacent notes are more probable in the two-octave than in the oneoctave range. Although this difference is not very great, it increases the relative pitch differences between reversals and nonreversals, which may be a critical factor.

A comparison of upper reversals, lower reversals, and nonreversals over a range of stimulus conditions permits an examination of the generality of any effects found.

\section{Method}

The experiment contained four parts, using pulse trains, square waves, a one-octave range, and a two-octave range. In each case, the three components, upper and lower contour reversals and nonreversals, were compared. Noise trials were evenly distributed among the three.

(a) Pulse trains. This experiment was run in five blocks of 20 trials, each block containing 12 noise and 8 signal trials.

(b) Square waves. The procedure was identical to (a), except that the stimuli were square waves instead of pulse trains.

(C) One octave. The pitch range of tunes was reduced to one octave. Because of this restriction, the method used to control the number of contour components within each tone sequence differed, as explained in the general method section. By minimizing the degree of control over the contour in this way, a sequence was allowed to contain anything from three to seven nonreversals (compared with five to seven with the previous method). All other details of procedure were identical to those above.

(d) Two oetaves. The contour of tone sequences was controlled in the same way as those of Experiment $2 \mathrm{c}$, but the notes were selected from a two-octave range.

\section{Results and Discussion}

Figure 5 illustrates the results across the four experimental conditions. A within- and between-subject analysis of variance compared results across the range of stimulus conditions. Newman-Keuls tests were used to make specific comparisons between pairs of means.

A three-way analysis of variance, condition $x$ contour component $x$ listeners, showed only one main effect, that of contour component $[F(2,152)=34.9$, $p<.0011$. There was no effect of condition and no interaction. Performance on upper reversals was significantly better than on both lower reversals and nonreversals, and lower reversals were superior to nonreversals. All differences were statistically significant at $\mathrm{p}<.01$.

The consistency of these results points to a more general (feature) notion of the role of reversals of contour. The pitch height hypothesis predicts that nonreversals would be better than lower reversals, and this was not found. The results of the previous

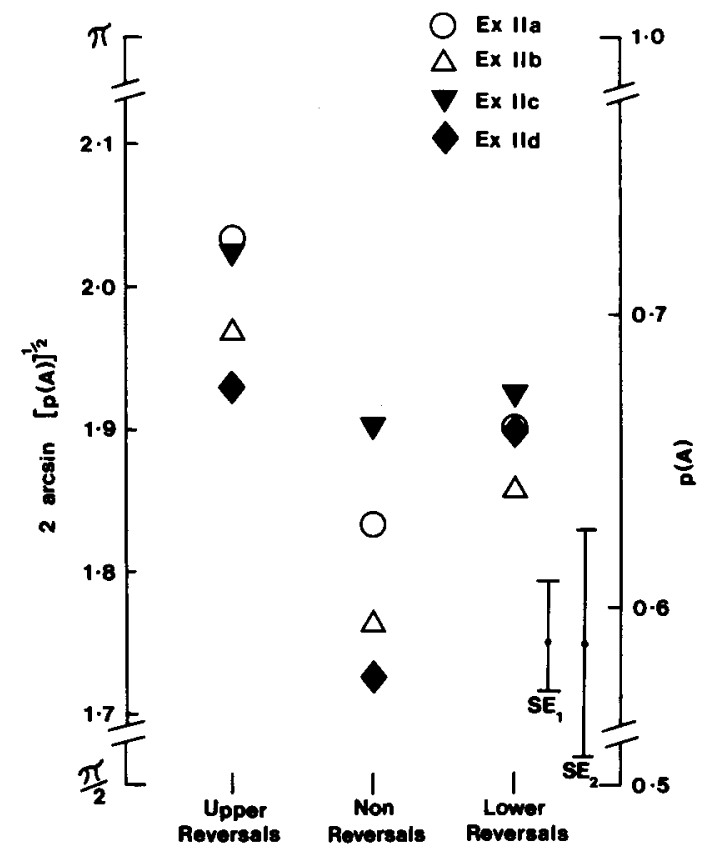

Figure 5. Results of Experiment 2a with pulse trains, Experiment $2 b$ with square waves, Experiment $2 c$ extending over one octave, and Experiment $2 d$ extending over two octaves. Means across listener are given and standard errors of within experimental condition $\left(\mathrm{SE}_{1}\right)$ and between conditions $\left(\mathrm{SE}_{2}\right)$.

experiment were also replicated, with upper reversals being significantly better than lower reversals. However, both types of reversals appear to contribute to the superior discrimination of reversals.

A comparison of the four experimental conditions excludes explanations in terms of differences in intensity and differences in relative and absolute pitch. Similar results were obtained using pulse trains and square waves and using a one- and a two-octave range. An interaction between note range and contour component would indicate that the perceptual salience of contour reversals, particularly upper reversals, could be due to relative pitch differences within the sequence or to absolute pitch height. Our results do not support this hypothesis. The lack of an interaction was also consistent with the conclusion of Experiment 1, that is, that the perceptual significance of parts of contour is not attributable to a form of auditory streaming. Furthermore, the analysis provides evidence that the two methods used to constrain contour did not produce perceptually significant differences in the sequences in terms of contour component effects.

The two experiments reported so far support a figural approach to contour which defines contour features, the reversals, or corners of the figure, as the perceptually salient aspects of the figure. These features seem to be common to melodic contours over a range of stimulus conditions. 


\section{EXPERIMENT 3}

The final experiment examined the extent to which the contour features found to be salient in novel melodies might be generalized to tunes with which the listener is made more familiar during the course of the experiment. The effect of transposition was also investigated.

In order to investigate the effect of transposition, it was necessary to modify the method of testing recognition memory. This was due to the difficulty of discriminating an exact transposition from a different tune with the same contour. Dowling and Fujitani (1971) found that listeners perform at chance level on this task. However, performance levels improve considerably if the first melody of a pair is held constant over a series of trials, allowing the listener to become familiar with, or learn, the melody (Watkins, 1982). This is true even when early trials, when the listener is unfamiliar with the melody, are mixed with later trials to calculate the discrimination score. Although this method reduced the set of tunes played to each listener, the task was made considerably easier.

The effects of this type of learning were separated from those of transposition by repeating the procedure with untransposed melodies. Higher scores were predicted for untransposed melodies than for transposed ones. However, the emphasis was placed on differences between contour components to see if contour features were salient in more familiar melodies.

\section{Method}

The experiment was broken down into two parts, using transposed and untransposed comparison melodies. Each part was divided into 10 blocks with 10 trials per block. The first tune of each trial was constant within a block, and listeners were required to compare the second tune with this tune on each trial. We wished to examine any differences in the salience of contour features that might be attributable to the familiarization inherent in this procedure. We did not establish the precise point at which the melody became familiar. It was sufficient to note that although the melody was unfamiliar to the listener at the beginning of a block of trials, repeated presentation encouraged the listener to learn the melody. Melodies were generated according to the constraints employed in Experiment 2d. A total of 10 different tunes were presented to each listener. Forty signal and 60 noise trials were used, with 20 noise trials per contour component (upper, lower, and nonreversal). The ratio of signal-to-noise trials in each block was varied randomly.

(a) Transposed. Listeners were required to discriminate between an exact transposition of the original tune and something slightly different (see general method) that had been transposed. The starting notes of both tunes were selected randomly on each trial, within certain limitations. For the first tune, notes were limited to middle $\mathrm{C} \pm 2$ semitones, whereas the transposed tune began within 5 semitones of middle $C$. Further restrictions prevented the second tune from starting on the same note. Transpositions ranged from 3 to 5 semitones up or down.

(b) Untransposed. The above procedure was repeated using a comparison melody that was not transposed and therefore started on the same note.

\section{Results and Discussion}

The results of this experiment, accompanied by those of Experiment 2, are shown in Figure 6. This enables a comparison to be made between the results obtained using novel melodies and those produced with the familiarization procedure. This was done using a three-way within- and between-subject analysis of variance. Again, the analysis was accompanied by Newman-Keuls multiple comparisons. The analysis of variance revealed two main effects, one of experimental condition $[F(2,57)=10.182, p<.001]$ and one of contour $[F(2,114)=15.732, p<.001]$, and also a significant interaction between contour and condition $[F(4,114)=5.120, p<.001]$.

The effect of experimental condition was due to the transposed condition (3a) and the untransposed comparison ( $3 \mathrm{~b})$, both of which employed the familiarization procedure. These produced significantly better results $(p<.01)$ than the condition without familiarization (2d), but did not differ from each other. The pattern of results due to contour component differed from that of the previous experiment. Although upper reversals were again discriminated more easily than lower reversals and nonreversals $(p<.01)$, there was no difference between lower reversals and nonreversals. The interaction between contour component and experimental condition appears to be due to the lack of a significant effect of contour component with transposed melodies $[\mathrm{F}(2,114)$

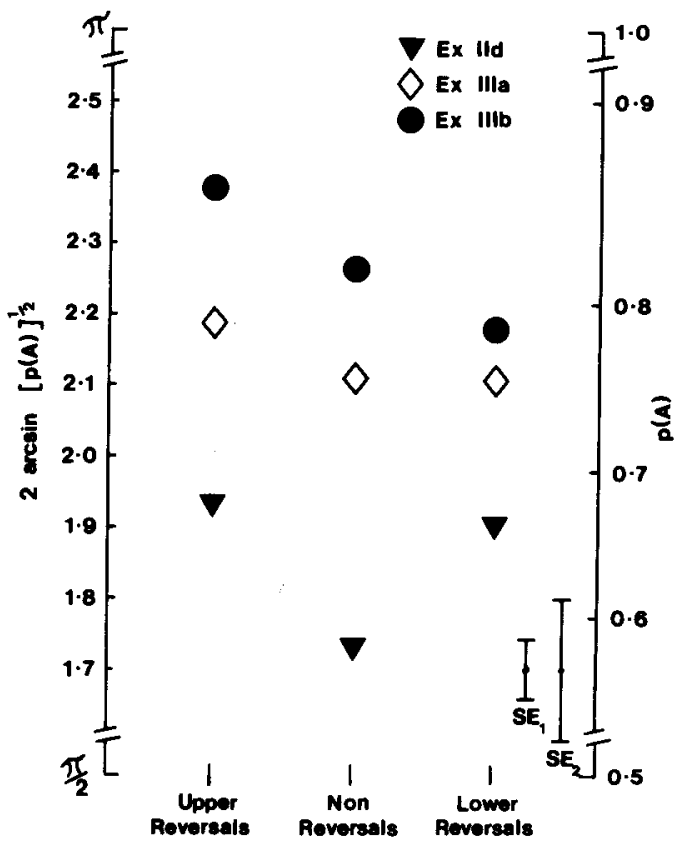

Figure 6. Results of Experiment 2d, untransposed, Experiment 3a, transposed with learning, and Experiment 3b, untransposed with learning. Means across listeners and the within experimental condition $\left(\mathrm{SE}_{1}\right)$ and between conditions $\left(\mathrm{SE}_{2}\right)$ standard errors. 
$=2.28, p>.05]$. Lower reversals were not salient in the familiarization procedure. Of the three contour components, the lowest discrimination scores were obtained with lower reversals.

The superior performance in the two conditions in which melodies were repeated demonstrates that listeners were indeed becoming familiar with the initially novel melodies. However, salient contour features did not emerge from here. We therefore conclude that the specific contour features important in the recognition of untransposed novel tunes (upper and lower reversals) do not generalize to more familiar tunes. Instead, upper reversals alone remain a salient feature, whereas lower reversals appear to lose their perceptual significance. There was a suggestion that transposition might have an additional influence, but this was not confirmed statistically.

It was not possible to disentangle the individual effects of transposition and familiarization. This requires the use of transposed melodies in the paradigm of Experiments 1 and 2. At the moment, we have established no means of obtaining performance levels above chance in this type of task.

\section{GENERAL DISCUSSION}

These experiments have revealed perceptually salient aspects of contour which may be interpreted as contour features. These features are the contour reversals and appear to be of a general nature: the relative salience of these pitch reversals is independent of the magnitude of pitch change or the type of waveform. In addition, upper reversals are more salient than lower reversals. Although these findings replicate over a range of stimulus conditions, they do not extend to transposed melodies and melodies with which listeners have been familiarized. In these cases, lower reversals are less salient than nonreversals, although upper reversals are, once again, the most salient. Therefore, although there are differences between contour components in the latter conditions, this might simply be due to a greater salience for the higher pitches.

Certain explanations for the results can be discounted: differences in absolute pitch, relative pitch, and auditory stream segregation could not account for our results.

One possible interpretation might be in terms of analogies between visual and auditory figures. The suggestion is that common organizational principles underlie the perceptual representation of both types of stimuli (see, e.g., Divenyi \& Hirsh, 1978; Heise \& Miller, 1951; Miller \& Heise, 1950; Ortmann, 1926). The findings of the present study show that reversals in melodic contour are in some ways similar to visual features as defined by Attneave (1954). That is, they seem to be treated as areas of high information value by the listener. Thus, in a single auditory presentation, listeners may be attempting to extract as much information as possible by aiming for the points of high information value, the corners, or reversals. These features may therefore be thought of as defining the shape of the tune, while the slopes (nonreversals) fill in the detail in between.

The observed reduction in the salience of these contour features, when the melodies become more familiar and are transposed, also has parallels with properties of visual figures. The perceptual salience of the corners of novel melodic contours might result from the type of global comparison envisaged in the first stage of Kroll and Hershenson's (1980) model of visual information processing. Here, only the outlines of figures are compared. However, repetition of the melody might allow time for the finer details to be filled in. Furthermore, transposing a melody changes some of its characteristics, such as absolute pitch range, so the task becomes more demanding and requires information on the exact relationships between notes. Therefore, with transposition and repetition of melodies, listeners are encouraged to perform the sort of additional analytic processing described in the second stage of Kroll and Hershenson's model. This would fill in the figural details of the contour, and so listeners would become less reliant upon the feature characteristics of the contour. This might explain some of the variations in feature salience for different tasks, although it does not explain the differential salience of the two global features, that is, upper versus lower reversals.

Another possibility is that listeners treat melodic contours and the pitch contours of speech in a similiar manner. There are similarities between the contour features of our novel melodies and the salient aspects of speech pitch contours. Furthermore, this correspondence between the perceptual importance of pitch reversals in melodic contour and changes in the fundamental frequency of speech has been observed in other experimental paradigms (Thomassen, 1982). The information conveyed by upper and lower reversals in intonation patterns may differ in nature. Although a rise in pitch, usually followed by a fall, gives prominence or stress (Lieberman, 1960), downward-pitch movement may signify a phrase boundary (Cooper \& Sorenson, 1977). Some workers have also attributed a differential salience to downward- and upward-pointing corners of the pitch contour in signifying the location of stress (Bolinger, 1958). Thus, these findings may provide a possible means of interpreting the greater salience of upper over lower reversals found in the present melody discrimination task.

These perceptual similarities between the pitch contours of melody and speech, and visual contours may have a common origin. However, in the absence of more closely comparable experiments and results 
with visual figures, it remains possible that the type of contour features the present experiments demonstrate is specific to the auditory modality. Nevertheless, there is no work on pitch contours of speech that would lead us to expect changes in feature salience when the contours are transposed and made more familiar.

Previously reported differences in the relative salience of components of an auditory sequence have been ascribed to a "major attentional emphasis toward relatively high frequency ... sounds" (Watson, Wroton, Kelly, \& Benbasset, 1975). The difference between upper and lower reversals and the differences between contour components with familiar transposed and untransposed melodies may be accounted for in this way. However, this cannot account for the general finding that reversals are more salient than nonreversals. This is because nonreversals are generally higher in pitch than the lower reversals.

Although earlier work on the recognition of melodies was concerned with the overall contour, there are certain characteristics which appear to be shared by both the overall contour and the contour components. Dowling and Fujitani (1971) found differences between untransposed and transposed melodies, as well as differences between novel and more familiar melodies. (This study used familiar folktunes, in contrast to the melodies of Experiment 3). Their results suggested that information on exact interval sizes was more important for recognition of familiar tunes than for unfamiliar melodies. For the latter, the melodic contour was much more significant. However, these two descriptions of melodic contour are readily distinguished: although the overall contour is useful in recognizing transposed melodies, the contour features of the present study are salient only with novel, untransposed comparisons.

The results reported in this paper demonstrate the general nature of contour features with regard to novel melodies, and encourage the view that these features may represent a fairly general perceptual phenomenon. The generality of the results may extend beyond the realm of music. Whether or not the salient perceptual features of auditory sequences can be translated into visual equivalents remains an empirical question (Dyson, 1983). Experiments on the recognition of visual figures, comparable to those used in this paper, may provide a more direct test of these visual analogies.

\section{SUMMARY AND CONCLUSIONS}

This paper found evidence for differential perceptual salience of aspects of melodic contour and interpreted these as features of an auditory figure. An analogy was made between melodic contour and visual figures. An alternative explanation was also discussed, drawing on similarities between the con- tour of melody and speech. The generality of these contour features was examined in detail.

The experiments demonstrated that: (1) contour reversals are more salient than nonreversals; (2) the perceptual salience of reversals is not due to a form of auditory stream segregation; (3) upper reversals are more salient than lower reversals (absolute pitch height, relative pitch differences, and intensity differences cannot account for these differences, although attentional differences might); (4) the perceptual salience of reversals extends over two types of stimuli, two pitch ranges, and two methods of controlling contour; and (5) more familiar melodies, with or without transposition, do not share perceptually salient characteristics with novel melodies.

In conclusion, melodic contour provides a figural description of novel tone sequences. Contour reversals serve as features contributing to a perceptual representation that gives a global outline of the melody to which further detail may be added.

\section{REFERENCES}

Attneave, F. (1954). Some informational aspects of visual perception. Psychological Review, 61, 183-193.

BAKER, M. A., \& LOEB, M. (1973). Implications of measurement of eye fixations for a psychophysics of form perception. Perception \& Psychophysics, 13, 185-192.

Bolinger, D. L. (1958). A theory of pitch accent in English. Word, 14, 109-149.

Bregman, A. S. (1978). Auditory streaming: Competition among alternative organizations. Perception \& Psychophysics, 23, 391-398.

Bregman, A. S., \& Aснiм, A. (1973). Visual stream segregation. Perception \& Psychophysics, 13, 451-454.

Bregman, A. S., \& Campeell, J. (1971). Primary auditory stream segregation and perception of order in rapid sequences of tones. Journal of Experimental Psychology, 89, 244-249.

Burns, E. M., \& W ARD, W. D. (1982). Intervals, scales and tuning. In D. Deutsch (Ed.), The psychology of music. New York: Academic Press.

Cohen, A., t'Hart, J. (1967). On the anatomy of intonation. Lingua, 19, 177-192.

Cooper, W. E., \& Sorenson, J. M. (1977). Fundamental frequency contours at syntactic boundaries. Journal of the Acoustical Society of America, 62, 683-692.

Davies, J. B., \& Jennings, J. (1977). Reproduction of familiar melodies and the perception of tonal sequences. Journal of the Acoustical Society of America, 61, 534-541.

DeUTsch, D. (1972). Octave generalization and tune recognition. Perception and Psychophysics, 11, 411-412.

DeuTsch, D. (1978a). Delayed pitch comparisons and the principle of proximity. Perception \& Psychophysics, 23, 227-230.

Deutsch, D. (1978b). The psychology of music. In E. C. Carterette \& M. P. Freedman (Eds.), Handbook of perception (Vol. 10). New York: Academic Press.

Divenyi, P. L., \& Hirsh, I. J. (1974). Identification of temporal order in a 3 tone sequence. Journal of the Acoustical Society of America, 56, 144-151.

DivenY1, P. L., \& Hirsh, I. J. (1975). The effect of blanking on the identification of temporal order in three-tone sequences. Perception \& Psychophysics, 17, 246-252.

Divenyi, P. L., \& Hirsh, I. J. (1978). Some figural properties of auditory patterns. Journal of the Acoustical Society of America, 64, 1369-1386.

Dowling, W. J. (1978). Scale and contour: Two components of a 
theory of memory for melodies. Psychological Review, 85, 341-354.

Dowling, W. J., \& Fujitani, D. S. (1971). Contour, interval and pitch recognition in memory for melodies. Journal of the Acoustical Society of America, 49, 524-531.

Dyson, M. C. (1983). Aspects of contour perception. Unpublished doctorai thesis, University of Reading, England.

Faure, G., Hinst, D. J., \& Chafcouloff, M. (1980). Rhythm in English: Isochronism, pitch and perceived stress. In L. R. Waugh \& C. H. Schooneveld (Eds.), The melody of language. Baltimore: University Park Press.

Fourcin, A. J., Rosen, S. M., Moore, B. C. J., Douer, E. E., Clarke, G. P., Dodson, H., \& Bannister, L. H. (1979). External electrical stimulation of the cochlea: Clinical, psychophysical, speech-perceptual and histological findings. British Journal of Audiology, 13, 85-107.

FRY, D. B. (1958). Experiments in the perception of stress. Language \& Speech, 1, 126-152.

Getry, D. J., Swets, J. A., Swets, J. R., \& Green, D. M. (1979). On the prediction of confusion matrices from similarity judgments. Perception \& Psychophysics, 26, 1-19.

Green, R. T., \& Countis, M. C. (1966). Information theory and figure perception: The metaphor that failed. Acta Psychologica, 25, 12-36.

Heise, G. A., \& Miller, G. A. (1951). An experimental study of auditory patterns. American Journal of Psychology, 64, 68-77.

Howard, J. H., \& Ballas, J. A. (1981). Feature selection in auditory perception. In D. J. Getty \& J. H. Howard (Eds.), Auditory and visual pattern recognition. Hillsdale, NJ: Erlbaum.

IDson, W. L., \& MAssaro, D. W. (1978). A bidimensional model of pitch in the recognition of melodies. Perception \& Psychophysics, 24, 551-556.

Kallman, H. J., \& Massaro, D. W. (1979). Tone chroma is functional in melody recognition. Perception \& Psychophysics, 26, 32-36.

Kroll, J. F., \& Hershenson, M. (1980). Two stages in visual matching. Canadian Journal of Psychology, 34, 49-61.

LERDAHL, F., \& JACKENDOFF, R. (1977). Toward a formal theory of tonal music. Journal of Music Theory, 21, 111-172.

Lieberman, P. (1960). Some acoustic correlates of word stress in American English. Journal of the Acoustical Society of America, 32, 451-454.

Longuet-Higains, H. C. (1976). Perception of melodies. Nature, 263, 646-653.

Longuet-Higains, H. C. (1978). The perception of music. Interdisciplinary Science Reviews, 3, 148-156.

Massaro, D. W., Kallman, H. J., \& Kelly, J. L. (1980). The role of tone height, melodic contour and tone chroma in melody recognition. Journal of Experimental Psychology: Human Learning \& Memory, 6, 77-90.

McNicol, D. (1972). A primer of signal detection theory. London: Allen \& Unwin.
Millen, G. A., \& Heise, G. A. (1950). The trill threshold. Journal of the Acoustical Society of America, 22, 637-638.

Morton, J., \& JAssem, W. (1965). Acoustic correlates of stress. Language of Speech, 8, 159-181.

Ortmann, O. (1926). On the melodic relativity of tones. Psychological Monographs, 35, 1-47.

OrTmann, O. (1933). Some tonal determinants of melodic memory. Journal of Educational Psychology, 24,454-467.

Rock, I., HAlPER, F., \& Clayton, T. (1972). The perception and recognition of complex figures. Cognitive Psychology, 3, 655-673.

Thomassen, J. M. (1982). Melodic accent: Experiments and a tentative model. Journal of the Acoustical Society of America, 71, 1596-1605.

van Noorden, L. P. A. S. (1975). Temporal coherence in the perception of tone sequences. Unpublished doctoral thesis, Technische Hogeschool, Eindhoven, Holland.

WARD, L. M., \& WEXLER, D. A. (1976). Levels of feature analysis in processing visual patterns. Perception, 5, 407-418.

Watkins, A. J. (1981a). Approximations to melodies. Acoustics Bulletin, 6(1), 16.

Watkins, A. J. (1981b). Tonal sequences, melody and music. Acoustics Bulletin, 6(2), 18.

Watkins, A. J. (1981c, Spring). Tonal sequences, melody and music. In Proceedings of the Institute of Acoustics (pp. 347-350). Edinburgh: Institute of Acoustics.

Watkins, A. J. (1982). 'Figural' and 'tonal' aspects of melody discrimination. Journal of the Acoustical Society of America, 72,545 .

Watson, C. S., Wroton, H. W., Kelly, W. J., \& Benbasset, C. A. (1975). Factors in the discrimination of tonal patterns. I. Component frequency, temporal position and silent intervals. Journal of the Acoustical Society of America, 57, 1175-1181.

\section{NOTES}

1. Except for Experiment 2c, in which a one-octave range around the keynote was used.

2. The addition or subtraction of a semitone avoids repeated successive notes.

3. The authors wish to thank an anonymous referee for raising this point.

4. All standard errors are within listener, as each data point is based on only one hit rate for each listener. A difference between means that is greater than 2 standard errors will be significant in a post hoc Student's $t$ test. When there are two factors within an experiment, the standard error of the interaction is used. When comparisons are made between experiments, the standard errors of both within and between experiments are given.

(Manuscript received August 18, 1983; revision accepted for publication March 8, 1984.) 